\title{
LONG WAVELENGTH INSTABILITY OF THE ABC-FLOWS
}

BY

\author{
A. LIBIN AND G. SIVASHINSKY *
}

The Levich Institute, The City College of New York, New' York, N. Y.

\begin{abstract}
Multiple-scale and spectral (Galerkin) techniques are employed to analyze the long-wave stability of a simple periodic helical flow at large Reynolds numbers. For a certain class of long-wave perturbations the corresponding mathematical problem admits an exact asymptotic solution, showing that the helical flow is always unstable.
\end{abstract}

I. Introduction. In recent years, significant attention has been attracted to the problem of long-wave instability of periodic flows [1-18]. It is believed that these systems may provide important insight into the phenomenon of inverse energy cascade and spontaneous formation of large-scale structures in 2-D and 3-D turbulence.

One of the simplest periodic flows capable (due to its intrinsic instability) of generating large scale structures is the so-called Arnold-Beltrami-Childress (ABC) flow

(i) $\mathbf{h}=V_{0}[A \sin (z / d)+B \cos (y / d), A \cos (z / d)+C \sin (x / d)$,

$$
B \sin (y / d)+C \cos (x / d)] .
$$

Here $V_{0}$ is a typical velocity, $2 \pi d$ is the space period of the flow.

In order to sustain the time-independent flow with (i) one must introduce an external force field

(ii) $\mathbf{f}=-\left(\frac{\nu}{d^{2}}\right) \mathbf{h}$

where $\nu$ is the kinematic viscosity. Previous studies have been focused primarily around two specific cases: $A=B=C=1$ (quasi-isotropic ABC-flow [12]) and $A=1, B=C=0$ (Beltrami flow) $[12,14,17]$. It was found that at finite Reynolds numbers $\left(R=V_{0} d / \nu \sim 1\right)$ the quasi-isotropic ABC-flow is stable relative to longwave perturbations [12], while the antisotropic Beltrami flow loses its stability at $R>\sqrt{2}[12,14]$. The long-wave instability at large Reynolds numbers has been studied only for the Beltrami flow [17].

The present work extends the long-wave stability analysis developed in [17] to the general case. It turns out that at $\mathrm{Re} \gg 1$ for a certain class of long-wave perturbations the corresponding mathematical problem admits an exact analytical solution, showing that $\mathrm{ABC}$-flow is always unstable-even in the quasi-isotropic case.

Received February 27. 1989.

* On leave from the School of Mathematical Sciences, Tel-Aviv University, Ramat-Aviv, Tel-Aviv, Israel.

(C) 1990 Brown University 
The paper is organized as follows. First, the limit of ideal nonviscous $(R=\infty)$ fluid is studied in detail (Sec. II). The results obtained are then extended to the case of large Reynolds numbers (Sec. III).

All the equations are written in the nondimensional form with $d, d / V_{0}, V_{0}$ taken as units of length, time, and velocity, correspondingly.

II. ABC-flow in the ideal fluid. Consider the Euler equation in the rotational form

$$
\frac{\partial}{\partial t}(\operatorname{rot} \mathbf{V})+\operatorname{rot}[\operatorname{rot} \mathbf{V} \times \mathbf{V}]=0
$$

where $\mathbf{V}=\left(V_{1}(x, y, z, t), V_{2}(x, y, z, t), V_{3}(x, y, z, t)\right)$

$$
\operatorname{div} \mathbf{V}=0 \text {. }
$$

Let $\mathbf{h}$ be the ABC-flow:

$$
\mathbf{h}=\left(\begin{array}{l}
A \sin z+B \cos y \\
A \cos z+C \sin x \\
B \sin y+C \cos x
\end{array}\right) .
$$

Since $\operatorname{rot} \mathbf{h}=\mathbf{h}, \mathbf{h}$ is a solution of the Euler equation (1). The linearization of Eq. (1) around $\mathbf{h}$ yields

$$
\left\{\begin{array}{l}
\frac{\partial}{\partial t}(\operatorname{rot} \mathbf{u})+\operatorname{rot}[\mathbf{h} \times(\mathbf{u}-\operatorname{rot} \mathbf{u})]=0 \\
\operatorname{div} \mathbf{u}=0
\end{array}\right.
$$

The main object of the present analysis is to verify the stability of the trivial solution $\mathbf{u}=0$ of Eq. (3).

The first step is the scaling transformation of Eqs. (3):

$$
\begin{gathered}
\mathbf{u}(x, y, z, t) \rightarrow \mathbf{u}(x, y, z, \xi, \eta, \theta, \tau), \\
\nabla_{x y z} \rightarrow \nabla_{x y z}+\varepsilon \nabla_{\xi \eta \theta} \quad(\varepsilon=\varepsilon x, \eta=\varepsilon y, \theta=\varepsilon z), \\
\partial_{t} \rightarrow \varepsilon \partial_{\tau} \quad(\tau=\varepsilon t) .
\end{gathered}
$$

Therefore

$$
\operatorname{rot}_{x y z} \rightarrow \operatorname{rot}_{x y z}+\varepsilon \operatorname{rot}_{\xi \eta \theta}
$$

and

$$
\operatorname{div}_{x y z} \rightarrow \operatorname{div}_{x y z}+\varepsilon \operatorname{div}_{\xi \eta \theta} .
$$

The rescaled equations ( 3 ) become

$$
\left.\begin{array}{l}
\varepsilon \frac{\partial}{\partial \tau}\left(\operatorname{rot}_{x y z} \mathbf{u}\right)+\varepsilon^{2} \frac{\partial}{\partial \tau}\left(\operatorname{rot}_{\xi \eta \theta}\right) \operatorname{rot}_{x y z}[\mathbf{h} \times(\mathbf{u}-\operatorname{rot} \mathbf{u})] \\
\quad-\operatorname{rot}_{x y z}\left[\mathbf{h} \times \operatorname{rot}_{\xi \eta \theta} \mathbf{u}\right]+\varepsilon \operatorname{rot}_{\xi \eta \theta}\left[\mathbf{h} \times\left(\mathbf{u}-\operatorname{rot}_{x y z} \mathbf{u}\right)\right] \\
\quad-\varepsilon^{2} \operatorname{rot}_{\xi \eta \theta}\left[\mathbf{h} \times \operatorname{rot}_{\xi \eta \theta} \mathbf{u}\right]=0, \\
\operatorname{div}_{x y z} \mathbf{u}=-\varepsilon \operatorname{div}_{\xi \eta \theta} \mathbf{u .}
\end{array}\right\}
$$

We are seeking solutions of (5) which are $2 \pi$-periodic in $x, y, z$. Hence, the 
integration of (5) from 0 to $2 \pi$ in $x, y$, and $z$ yields

$$
\begin{aligned}
\varepsilon \frac{\partial}{\partial \tau} & \int_{0}^{2 \pi} \int_{0}^{2 \pi} \int_{0}^{2 \pi} \operatorname{rot}_{\xi \eta \theta} \mathbf{u} d x d y d z \\
& +\int_{0}^{2 \pi} \int_{0}^{2 \pi} \int_{0}^{2 \pi} \operatorname{rot}_{\xi \eta \theta}\left[\mathbf{h} \times\left(\mathbf{u}-\operatorname{rot}_{x y z} \mathbf{u}\right)\right] d x d y d z \\
& -\varepsilon \int_{0}^{2 \pi} \int_{0}^{2 \pi} \int_{0}^{2 \pi} \operatorname{rot}_{\xi \eta \theta}\left[\mathbf{h} \times \operatorname{rot}_{\xi \eta \theta} \mathbf{u}\right] d x d y d z=0
\end{aligned}
$$

We now consider $\varepsilon$ as a small parameter and seek a solution of the system (5), (6) in the form of an asymptotic expansion in powers of $\varepsilon$ :

$$
\mathbf{u}(x, y, z, \xi, \eta, \theta, \tau)=\mathbf{u}_{0}+\varepsilon \mathbf{u}_{1}+\varepsilon^{2} \mathbf{u}_{2}+\cdots .
$$

Thus for the $\varepsilon^{0}$-approximation of (5) we obtain

$$
\left.\begin{array}{l}
\operatorname{rot}_{x y z}\left[h \times\left(\mathbf{u}_{0}-\operatorname{rot}_{x y z} \mathbf{u}_{0}\right)=0\right. \\
\operatorname{div}_{x y z} \mathbf{u}_{0}=0 .
\end{array}\right\}
$$

Consider the following set of vectors:

$$
\begin{array}{ll}
\mathbf{e}_{0}=\left(\begin{array}{c}
\sin z \\
\cos z \\
0
\end{array}\right), & \mathbf{f}_{0}=\left(\begin{array}{c}
\cos y \\
0 \\
\sin y
\end{array}\right), \quad \mathbf{g}_{0}=\left(\begin{array}{c}
0 \\
\sin x \\
\cos x
\end{array}\right), \\
\mathbf{e}_{1}=\left(\begin{array}{c}
\cos z \\
-\sin z \\
0
\end{array}\right), & \mathbf{f}_{0}=\left(\begin{array}{c}
-\sin y \\
0 \\
\cos y
\end{array}\right), \quad \mathbf{g}_{1}=\left(\begin{array}{c}
0 \\
\cos x \\
-\sin y
\end{array}\right) .
\end{array}
$$

Vectors (8) from the orthogonal basis of the proper subspace of the $\operatorname{rot}_{x y z}$ operator in the Hilbert space with eigenvalue 1. we should seek $u_{0}$ in the following form

$$
\begin{aligned}
\mathbf{u}_{0}(x, y, z, \xi, \eta, \theta, \tau)= & \alpha_{0}(\eta, \theta, \tau) \mathbf{g}_{0}+\beta_{0}(\xi, \theta, \tau) \mathbf{f}_{0}+\gamma_{0}(\xi, \eta, \tau) \mathbf{e}_{0} \\
& +\alpha_{1}(\eta, \theta, \tau) \mathbf{g}_{1}+\beta_{1}(\xi, \theta, \tau) \mathbf{f}_{1}+\gamma_{1}(\xi, \eta, \tau) \mathbf{e}_{1}+\mathbf{G},
\end{aligned}
$$

where $\mathbf{G}=\left[G_{1}(\xi, \eta, \theta, \tau), G(\xi, \eta, \theta, \tau), G_{3}(\xi, \eta, \theta, \tau)\right]$. Actually $\mathbf{h}=A \mathbf{e}_{0}+$ $B \mathbf{f}_{0}+C \mathbf{g}_{0}$. The substitution of (9) into (7) yields

$$
A G_{3} \mathbf{e}_{1}+B G_{2} \mathbf{f}_{1}+C G \mathbf{e}_{1} \equiv 0 .
$$

Hence

$$
\mathbf{G} \equiv 0, \quad \text { if } A \neq 0, B \neq 0, C \neq 0 .
$$

For $\mathbf{u}_{0}$ in the form (9), the integral relationship (6) for $\varepsilon^{0}$-approximation is automatically satisfied. In order to determine the coefficients in (9) we have to pass to the $\varepsilon^{1}$-approximation in (5) and $\varepsilon^{2}$-approximation in (6),

$$
\begin{aligned}
& \left.\frac{\partial}{\partial \tau}\left(\operatorname{rot}_{x y z} \mathbf{u}_{0}\right)-\operatorname{rot}_{x y z}\left[\mathbf{h} \times \operatorname{rot}_{\xi \eta \theta} \mathbf{u}_{0}\right]+\operatorname{rot}_{\xi \eta \theta}\left[\mathbf{h} \times\left(\mathbf{u}_{0}-\operatorname{rot}_{x y z} \mathbf{u}_{0}\right)\right]\right) \\
& =-\operatorname{rot}_{x y z}\left[\mathbf{h} \times\left(\mathbf{u}_{1}-\operatorname{rot}_{x y z} \mathbf{u}_{1}\right)\right], \\
& \operatorname{div}_{x y z} \mathbf{u}_{1}=-\operatorname{div}_{\xi \eta \theta} \mathbf{u}_{0}, \\
& \operatorname{div}_{x y z} \mathbf{u}_{2}=\operatorname{div}_{\xi \eta \theta} \mathbf{u}_{1} \\
& \frac{\partial}{\partial \tau} \int_{0}^{2 \pi} \int_{0}^{2 \pi} \int_{0}^{2 \pi} \operatorname{rot}_{\xi \eta \theta}\left(\mathbf{u}_{1}\right) d x d y d z \\
& +\int_{0}^{2 \pi} \int_{0}^{2 \pi} \int_{0}^{2 \pi} \operatorname{rot}_{\xi \eta \theta}\left[\mathbf{h} \times\left(\mathbf{u}_{2}-\operatorname{rot}_{x y z} \mathbf{u}_{2}\right)\right] d x d y d z \\
& =\int_{0}^{2 \pi} \int_{0}^{2 \pi} \int_{0}^{2 \pi} \operatorname{rot}_{\xi \eta \theta}\left[h \times \operatorname{rot}_{\xi \eta \theta} \mathbf{u}_{1}\right] d x d y d z \text {. }
\end{aligned}
$$


Set

$$
\mathbf{u}_{1}=\left[V_{1}(x, y, z, \xi, \eta, \theta, \tau), V_{2}(x, y, z, \xi, \eta, \theta, \tau), V_{3}(x, y, z, \xi, \eta, \theta, \tau)\right] .
$$

Thus, in view of $(9)$, the second equation in (10) is

$$
\begin{aligned}
\frac{\partial V_{1}}{\partial x}+\frac{\partial V_{2}}{\partial y}+\frac{\partial V_{3}}{\partial z}= & \left(\frac{\partial \gamma_{1}}{\partial \eta}-\frac{\partial \gamma_{0}}{\partial \xi}\right) \sin z-\left(\frac{\partial \gamma_{1}}{\partial \xi}+\frac{\partial \gamma_{0}}{\partial \eta}\right) \cos z \\
& +\left(\frac{\partial \beta_{1}}{\partial \xi}-\frac{\partial \beta_{0}}{\partial \theta}\right) \sin y-\left(\frac{\partial \beta_{1}}{\partial \theta}+\frac{\partial \beta_{0}}{\partial \xi}\right) \cos y \\
& +\left(\frac{\partial \alpha_{1}}{\partial \theta}-\frac{\partial \alpha_{0}}{\partial \eta}\right) \sin x-\left(\frac{\partial \alpha_{1}}{\partial \eta}+\frac{\partial \alpha_{0}}{\partial \theta}\right) \cos x
\end{aligned}
$$

Since $V_{1}, V_{2}, V_{3}$ are $2 \pi$-periodic in $x, y, z$ we get

$$
\begin{aligned}
& \mathbf{u}_{1}=\left(\begin{array}{l}
\left(\frac{\partial \alpha_{0}}{\partial \eta}-\frac{\partial \alpha_{1}}{\partial \theta}\right) \cos x-\left(\frac{\partial \alpha_{1}}{\partial \eta}+\frac{\partial \alpha_{0}}{\partial \theta}\right) \sin x \\
\left(\frac{\partial \beta_{0}}{\partial \theta}-\frac{\partial \beta_{1}}{\partial \xi}\right) \cos y-\left(\frac{\partial \beta_{0}}{\partial \xi}+\frac{\partial \beta_{1}}{\partial \theta}\right) \sin y \\
\left(\frac{\partial \gamma_{0}}{\partial \xi}-\frac{\partial i_{1}}{\partial \eta}\right) \cos z-\left(\frac{\partial \dot{\gamma}_{0}}{\partial \eta}+\frac{\partial \dot{\gamma}_{1}}{\partial \xi}\right) \sin z
\end{array}\right)+\left(\begin{array}{l}
\delta_{1}(\xi, \eta, \theta, \tau) \\
\delta_{2}(\xi, \eta, \theta, \tau) \\
\delta_{3}(\xi, \eta, \theta, \tau)
\end{array}\right)+\mathbf{w}_{1} \\
& =\mathbf{V}_{1}+\gamma+\mathbf{w}_{1} \text {, }
\end{aligned}
$$

where $\operatorname{div}_{x y z} \mathbf{w}_{1}=0, \operatorname{div}_{\xi \eta \theta} \delta=0$, and the projection of $\mathbf{w}$ is orthogonal in the Hilbert space to the subspace of constant (in $x, y, z$ ) vectors, and on the subspace of vectors with zero vorticity is null.

Note that the first two vectors in the right-hand side of (11) ( $\mathbf{v}$ and $\boldsymbol{\delta}$ ) belong exactly to these subspaces. In view of the specific choice of coefficients in (9), direct evaluation of $\operatorname{rot}_{\xi \eta \theta} \mathbf{u}_{0}$ yields

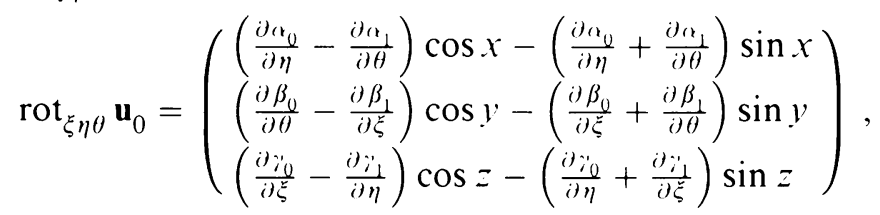

i.e.,

$$
\operatorname{rot}_{\xi \eta \theta} \mathbf{u}_{0}=\mathbf{V}_{1}
$$

or

$$
\mathbf{u}_{1}=\operatorname{rot}_{\xi \eta \theta} \mathbf{u}_{0}+\boldsymbol{\delta}+\mathbf{w}_{1} .
$$

Hence $\operatorname{rot}_{x y z}\left[\mathbf{h} \times \operatorname{rot}_{\xi \eta \theta} \mathbf{u}_{0}\right]=\operatorname{rot}_{x y z}\left[\mathbf{h} \times \mathbf{V}_{1}\right]$. On the other hand, in view of (12)

$$
\frac{\partial}{\partial \tau}\left(\operatorname{rot}_{x y z} u_{0}\right)+\operatorname{rot}_{x y z}[\mathbf{h} \times \boldsymbol{\delta}]=0 .
$$

Direct evaluation yields:

$$
\begin{aligned}
{[\mathbf{h} \times \boldsymbol{\delta}]=} & A \delta_{3} \mathbf{e}_{1}+B \delta_{2} \mathbf{f}_{1}+C \delta_{1} \mathbf{g}_{1} \\
& +\delta_{3}\left(\begin{array}{c}
C \sin x \\
-B \cos y \\
0
\end{array}\right)+\delta_{2}\left(\begin{array}{c}
-C \cos x \\
0 \\
A \sin z
\end{array}\right)+\delta_{1}\left(\begin{array}{c}
0 \\
B \sin y \\
-A \cos x
\end{array}\right) .
\end{aligned}
$$

Last three vectors in this expansion are of zero vorticity. Thus

$$
\operatorname{rot}_{x y z}[\mathbf{h} \times \boldsymbol{\delta}]=A \delta_{3} \mathbf{e}_{1}+B \delta_{2} \mathbf{f}_{1}+C \delta_{1} \mathbf{g}_{1} .
$$


In view of (9) we get from (10a)

$$
\begin{aligned}
& \frac{\partial \gamma_{0}}{\partial \tau} \mathbf{e}_{0}+\frac{\partial \beta_{0}}{\partial \tau} \mathbf{f}_{0}+\frac{\partial \alpha_{0}}{\partial \tau} \mathbf{g}_{0}+\left(\frac{\partial \beta_{1}}{\partial \tau}+A \delta_{3}\right) \mathbf{e}_{1} \\
& \quad+\left(\frac{\partial \beta_{1}}{\partial \tau}+B \delta_{2}\right) \mathbf{f}_{1}+\left(\frac{\partial \gamma_{1}}{\partial \tau}+C \delta_{1}\right) \mathbf{g}_{1}=0 .
\end{aligned}
$$

$\mathrm{Or}$

$$
\begin{array}{ccc}
\frac{\partial \alpha_{0}}{\partial \tau}=0 ; & \frac{\partial \beta_{0}}{\partial \tau}=0 ; & \frac{\partial \gamma_{1}}{\partial \tau}=0 ; \\
\frac{\partial \alpha_{0}}{\partial \tau}=-C \delta_{1} ; & \frac{\partial \beta_{0}}{\partial \tau}=-B \delta_{2} ; & \frac{\partial \gamma_{1}}{\partial \tau}=-A \delta_{3} .
\end{array}
$$

In the seeking of solutions of (5) which grow in time, $\alpha_{0}, \beta_{0}$, and $\gamma_{0}$ are of no interest for our purpose.

Hence $\mathbf{u}_{0}=\alpha_{1}(\eta, \theta, \tau) \mathbf{g}_{1}+\beta(\xi, \theta, \tau) \mathbf{f}_{1}+\gamma_{1}(\xi, \eta, \tau) \mathbf{e}_{1}$ and

$$
\mathbf{V}_{1}=-\left(\begin{array}{l}
\frac{\partial \alpha_{1}}{\partial \theta} \cos x+\frac{\partial \alpha_{1}}{\partial \eta} \sin x \\
\frac{\partial \beta_{1}}{\partial \xi} \cos y+\frac{\partial \beta_{1}}{\partial \theta} \sin y \\
\frac{\partial \gamma_{1}}{\partial \eta} \cos z+\frac{\partial \eta_{1}}{\partial \xi} \sin z
\end{array}\right)
$$

Set $\mathbf{w}_{1}=\lambda_{1} \mathbf{g}_{1}+\lambda_{2} \mathbf{f}_{1}+\lambda_{3} \mathbf{e}_{1}$. Clearly $\operatorname{rot}_{x y z} \mathbf{w}_{1}=\mathbf{w}_{1}$. Thus

$$
\operatorname{rot}_{\xi \eta \theta} \mathbf{w}_{1}=\left(\begin{array}{c}
\frac{\partial \lambda_{1}}{\partial \eta} \cos x+\frac{\partial \lambda_{1}}{\partial \theta} \sin x \\
\frac{\partial \lambda_{1}}{\partial \theta} \cos y+\frac{\partial \lambda_{1}}{\partial \xi} \sin y \\
\frac{\partial \lambda_{1}}{\partial \xi} \cos z+\frac{\partial \lambda_{1}}{\partial \eta} \sin z
\end{array}\right)+\left(\begin{array}{c}
\frac{\partial \lambda_{3}}{\partial \theta} \sin z \\
\frac{\partial \lambda_{3}}{\partial \theta} \cos z \\
0
\end{array}\right)+\left(\begin{array}{c}
\frac{\partial \lambda_{2}}{\partial \eta} \cos y \\
0 \\
\frac{\partial \lambda_{2}}{\partial \eta} \sin y
\end{array}\right)+\left(\begin{array}{c}
0 \\
\frac{\partial \lambda_{2}}{\partial \xi} \sin x \\
\frac{\partial \lambda_{2}}{\partial \xi} \cos x
\end{array}\right) \text {. }
$$

On the other hand

$$
\begin{aligned}
\operatorname{div}_{x y z} \mathbf{u}_{2}= & -\operatorname{div}_{\xi \eta \theta} \mathbf{u}_{1} \\
= & -\operatorname{div}_{\xi \eta \theta} \mathbf{v}_{1}-\operatorname{div}_{\xi \eta \theta} \boldsymbol{\delta}-\operatorname{div}_{\xi \eta \theta} \mathbf{w}_{1} \\
= & -\operatorname{div}_{\xi \eta \theta} \mathbf{w}_{1} \\
= & \frac{\partial \lambda_{1}}{\partial \theta} \sin x-\frac{\partial \lambda_{1}}{\partial \eta} \cos x+\frac{\partial \lambda_{2}}{\partial \xi} \sin y \\
& -\frac{\partial \lambda_{2}}{\partial \theta} \cos y+\frac{\partial \lambda_{3}}{\partial \eta} \sin z-\frac{\partial \lambda_{3}}{\partial \xi} \cos z
\end{aligned}
$$

Therefore

$$
\mathbf{u}_{2}=-\left(\begin{array}{l}
\frac{\partial \lambda_{1}}{\partial \theta} \cos x+\frac{\partial \lambda_{1}}{\partial \eta} \sin x \\
\frac{\partial \lambda_{1}}{\partial \xi} \cos y+\frac{\partial \lambda_{1}}{\partial \theta} \sin y \\
\frac{\partial \lambda_{1}}{\partial \eta} \cos z+\frac{\partial \lambda_{1}}{\partial \xi} \sin z
\end{array}\right)+\boldsymbol{\delta}_{2}+\mathbf{w}_{2}=\mathbf{V}_{2}+\boldsymbol{\delta}_{2}+w_{2},
$$

where $\operatorname{div}_{x y z} \mathbf{w}_{2}=0, \operatorname{div}_{\xi \eta \theta} \boldsymbol{\delta}_{2}=0$. Hence, $\operatorname{rot}_{\xi \eta \theta} \mathbf{w}_{1}=\mathbf{V}_{2}+\boldsymbol{\zeta}$, where

$$
\int_{0}^{2 \pi} \int_{0}^{2 \pi} \int_{0}^{2 \pi}[\mathbf{h} \times \zeta] d x d y d z=0
$$

On the other hand

$$
\int_{0}^{2 \pi} \int_{0}^{2 \pi} \int_{0}^{2 \pi}\left[\mathbf{h} \times\left(\mathbf{w}_{2}-\operatorname{rot}_{x y z} \mathbf{w}_{2}\right)\right] d x d y d z=0,
$$


since $\mathbf{w}_{2}-\operatorname{rot}_{x y z} \mathbf{w}_{2}$ does not have any projection in the Hilbert space on the subspace of the gradients of the scalar functions (proper subspace of the rot $x y=$ operator with the eigenvalue 0 -the kernel of the operator). Hence, due to (21)

$$
\begin{aligned}
\int_{0}^{2 \pi} & \int_{0}^{2 \pi} \int_{0}^{2 \pi}\left[\mathbf{h} \times\left(\mathbf{u}_{2}-\operatorname{rot}_{x y z} \mathbf{u}_{2}\right)\right] d x d y d z \\
& =\int_{0}^{2 \pi} \int_{0}^{2 \pi} \int_{0}^{2 \pi}\left[\mathbf{h} \times \mathbf{V}_{2}\right] d x d y d z \\
& =\int_{0}^{2 \pi} \int_{0}^{2 \pi} \int_{0}^{2 \pi}\left[\mathbf{h} \times \operatorname{rot}_{\xi \eta \theta} \mathbf{w}_{1}\right] d x d y d z .
\end{aligned}
$$

Hence, the integral in the right-hand side of the integral relationship in (10) is

$$
\begin{aligned}
& \int_{0}^{2 \pi} \int_{0}^{2 \pi} \int_{0}^{2 \pi}\left[\mathbf{h} \times \operatorname{rot}_{\xi \eta \theta} \mathbf{u}_{1}\right] d x d y d z \\
&=\int_{0}^{2 \pi} \int_{0}^{2 \pi} \int_{0}^{2 \pi}\left[\mathbf{h} \times \operatorname{rot}_{\xi \eta \theta} \mathbf{V}_{1}\right] d x d y d z \\
& \quad+\int_{0}^{2 \pi} \int_{0}^{2 \pi} \int_{0}^{2 \pi}\left[\mathbf{h} \times \operatorname{rot}_{\xi \eta \theta} \mathbf{w}_{1}\right] d x d y d z \\
& \quad+\int_{0}^{2 \pi} \int_{0}^{2 \pi} \int_{0}^{2 \pi}\left[\mathbf{h} \times \boldsymbol{\delta}_{1}\right] d x d y d z \\
&=\int_{0}^{2 \pi} \int_{0}^{2 \pi} \int_{0}^{2 \pi}\left[\mathbf{h} \times \operatorname{rot}_{\xi \eta \theta} \mathbf{v}_{1}\right] d x d y d z \\
&+\int_{0}^{2 \pi} \int_{0}^{2 \pi} \int_{0}^{2 \pi}\left[\mathbf{h} \times\left(\mathbf{u}_{2}-\operatorname{rot}_{x y z} \mathbf{u}_{2}\right)\right] d x d y d z
\end{aligned}
$$

Thus, the integral relationship (in 10) is actually

$$
\frac{\partial}{\partial \tau} \int_{0}^{2 \pi} \int_{0}^{2 \pi} \boldsymbol{\delta} d x d y d z=\int_{0}^{2 \pi} \int_{0}^{2 \pi} \int_{0}^{2 \pi}\left[\mathbf{h} \times \operatorname{rot}_{\xi \eta \theta} \mathbf{V}_{1}\right] d x d y d z
$$

or

$$
\frac{\partial}{\partial \tau} \int_{0}^{2 \pi} \int_{0}^{2 \pi} \int_{0}^{2 \pi} \boldsymbol{\delta} d x d y d z=\int_{0}^{2 \pi} \int_{0}^{2 \pi} \int_{0}^{2 \pi}\left[\mathbf{h} \times\left(\operatorname{rot}_{\xi \eta \theta} \operatorname{rot}_{\xi \eta \theta} \mathbf{u}_{0}\right] d x d y d z\right.
$$

since $\mathbf{V}_{1}=\operatorname{rot}_{\xi \eta \theta} \mathbf{u}_{0}$.

In view of (12), we get $\left(\alpha_{0} \equiv \beta_{0} \equiv \gamma_{0} \equiv 0\right)$

Hence

$$
\operatorname{rot}_{\xi \eta \theta} \mathbf{V}_{1}=\left(\begin{array}{l}
\frac{\partial^{2} \beta_{1}}{\partial \xi \partial \theta} \cos y+\frac{\partial^{2} \beta_{1}}{\partial \theta^{2}} \sin y-\frac{\partial^{2} \eta_{1}}{\partial \eta^{2}} \cos z-\frac{\partial^{2} \eta_{1}}{\partial \theta \partial \xi} \sin z \\
\frac{\partial^{2} \eta_{1}}{\partial \xi \partial \eta} \cos z+\frac{\partial^{2} \eta_{1}}{\partial \xi^{2}} \sin z-\frac{\partial^{2} \alpha_{1}}{\partial \theta^{2}} \cos x-\frac{\partial^{2} \alpha_{1}}{\partial \eta \partial \xi} \sin x \\
\frac{\partial^{2} \alpha_{1}}{\partial \eta \partial \theta} \cos x+\frac{\partial^{2} \alpha_{1}}{\partial \eta^{2}} \sin x-\frac{\partial^{2} \beta_{1}}{\partial \xi^{2}} \cos y-\frac{\partial^{2} \alpha_{1}}{\partial \theta \partial \xi} \sin y
\end{array}\right) .
$$

$$
\left[\mathbf{h} \times \operatorname{rot}_{\xi \eta \theta} \mathbf{V}_{1}\right]=\left(\begin{array}{c}
\frac{c}{2}\left(\frac{\partial^{2} \alpha_{1}}{\partial \eta^{2}}+\frac{\partial^{2} \alpha_{1}}{\partial \theta^{2}}\right) \\
\frac{B}{2}\left(\frac{\partial^{2} \beta_{1}}{\partial \xi^{2}}+\frac{\partial^{2} \beta_{1}}{\partial \theta^{2}}\right) \\
\frac{A}{2}\left(\frac{\partial^{2} \eta_{1}}{\partial \xi^{2}}+\frac{\partial^{2} \eta_{1}}{\partial \eta^{2}}\right)
\end{array}\right)+\cdots,
$$


where the truncated terms vanish after integration. Thus, after integration (25) becomes

$$
\begin{gathered}
\frac{\partial \delta_{1}}{\partial \tau}=\frac{C}{2}\left(\frac{\partial^{2} \alpha_{1}}{\partial \theta^{2}}+\frac{\partial^{2} \alpha_{1}}{\partial \eta^{2}}\right), \\
\frac{\partial \delta_{2}}{\partial \tau}=\frac{B}{2}\left(\frac{\partial^{2} \beta_{1}}{\partial \xi^{2}}+\frac{\partial^{2} \beta_{1}}{\partial \theta^{2}}\right), \\
\frac{\partial \delta_{3}}{\partial \tau}=\frac{A}{2}\left(\frac{\partial^{2} \gamma_{1}}{\partial \eta^{2}}+\frac{\partial^{2} \gamma_{1}}{\partial \xi^{2}}\right) .
\end{gathered}
$$

In fact, $\operatorname{rot}_{\xi \eta \theta}$ of both sides of (25) are equal. Thus, in principle, one can add $\operatorname{grad} F(\xi, \eta, \theta, \tau)$ to the right-hand side. However, $\operatorname{divgrad} F \equiv 0$ or $\Delta_{\xi \eta \theta} F=0$. Since we are seeking bounded solutions of this equation in the whole space, $F \equiv 0$.

Thus, (17) and (28) form a closed system of equations, reducible to three independent subsystems

$$
\begin{aligned}
& \frac{\partial \alpha_{1}}{\partial \tau}=-C \delta_{1}, \\
& \frac{\partial \delta_{1}}{\partial \tau}=\frac{C}{2}\left(\frac{\partial^{2} \alpha_{1}}{\partial \eta^{2}}+\frac{\partial^{2} \alpha_{1}}{\partial \theta^{2}}\right), \\
& \frac{\partial \beta_{1}}{\partial \tau}=-B \delta_{1}, \\
& \frac{\partial \delta_{1}}{\partial \tau}=\frac{B}{2}\left(\frac{\partial^{2} \beta_{1}}{\partial \xi^{2}}+\frac{\partial^{2} \beta_{1}}{\partial \theta^{2}}\right), \\
& \frac{\partial \gamma_{1}}{\partial \tau}=-A \delta_{3}, \\
& \frac{\partial \delta_{3}}{\partial \tau}=\frac{A}{2}\left(\frac{\partial^{2} \gamma_{1}}{\partial \xi^{2}}+\frac{\partial^{2} \gamma_{1}}{\partial \theta^{2}}\right) .
\end{aligned}
$$

Differentiation of the second equation in (2) with respect to $\tau$ and the substitution of $-C \delta_{1}$, instead of $\delta \alpha_{1} / \partial \tau$ in the new equation, yields

$$
\frac{\partial^{2} \delta_{1}}{\partial \tau^{2}}=-\frac{C^{2}}{2}\left(\frac{\delta^{2} \delta_{1}}{\partial \eta^{2}}+\frac{\delta^{2} \delta_{1}}{\partial \theta^{2}}\right)
$$

Similarly

$$
\begin{aligned}
& \frac{\partial^{2} \delta_{2}}{\partial \tau^{2}}=-\frac{B^{2}}{2}\left(\frac{\partial^{2} \delta_{2}}{\partial \xi^{2}}+\frac{\partial^{2} \delta_{2}}{\partial \theta_{2}}\right) \\
& \frac{\partial^{2} \delta_{2}}{\partial \tau^{2}}=-\frac{A^{2}}{2}\left(\frac{\partial^{2} \delta_{3}}{\partial \xi^{2}}+\frac{\partial^{2} \delta_{3}}{\partial \theta^{2}}\right)
\end{aligned}
$$


Hence, the corresponding dispersion relations are

$$
\begin{aligned}
& \omega_{1}^{2}=\frac{C^{2}}{2}\left(l^{2}+m^{2}\right), \\
& \omega_{2}^{2}=\frac{B^{2}}{2}\left(k^{2}+m^{2}\right), \\
& \omega_{3}^{2}=\frac{A^{2}}{2}\left(k^{2}+l^{2}\right) .
\end{aligned}
$$

Hence, if $A \neq 0, B \neq 0, C \neq 0$ there are three exponentially growing solutions which are linearly independent.

It is worth noting that the constructed solutions are the exact solutions of the system (10). ${ }^{\dagger}$

The case of the Beltrami flow is of special interest to us. For this flow the system (29.1-29.3) contains a closed subsystem

$$
\left\{\begin{array}{l}
\frac{\partial \eta_{1}}{\partial \tau}=-A \delta_{3} \\
\frac{\partial \delta_{3}}{\partial \tau}=\frac{A}{2}\left(\frac{\partial^{2} \eta_{1}}{\partial \dot{\xi}^{2}}+\frac{\partial^{2} \ddot{\eta}_{1}}{\partial \eta^{2}}\right)
\end{array}\right.
$$

This means, that we may consider the solutions dependent on $z$ only. The corresponding solution is

$$
\mathbf{u}_{0}+\varepsilon \mathbf{u}_{1}=\exp \left[i(k \xi+\mid \eta)+|A| \sqrt{\frac{k^{2}+l^{2}}{2} \tau}\right]\left(\begin{array}{c}
\cos z \\
-\sin z \\
i \varepsilon(l \cos z+k \sin z)-\varepsilon \sqrt{\frac{k^{2}+l^{2}}{2}}
\end{array}\right) .
$$

III. ABC-flow in viscouse liquid at large Reynolds numbers. Consider the NavierStokes equation in the rotational form

$$
\left\{\begin{array}{l}
\frac{\partial}{\partial t}(\operatorname{rot} \mathbf{V})+\operatorname{rot}[\operatorname{rot} \mathbf{V} \times \mathbf{V}]=\frac{1}{R} \operatorname{rot}(\Delta \mathbf{V})+\frac{1}{R} \mathbf{F}, \\
\operatorname{div} \mathbf{V}=0 .
\end{array}\right.
$$

Let $\mathbf{F}$ be a vector-function such that (35) is an identity for

$$
\begin{aligned}
\mathbf{V} & =\mathbf{h} \\
& =[A \sin z+B \cos y, A \cos z+C \sin x, B \sin y+C \cos x] \\
& =A \mathbf{e}_{0}+B \mathbf{f}_{0}+C \mathbf{g}_{0} .
\end{aligned}
$$

The linearization of (35) around $\mathbf{V}=\mathbf{h}$ yields

$$
\left\{\begin{array}{l}
\frac{\partial}{\partial t}(\operatorname{rot} \mathbf{u})+\operatorname{rot}[\mathbf{h} \times(\mathbf{u}-\operatorname{rot} \mathbf{u})]=\frac{1}{R} \operatorname{rot}(\Delta \mathbf{u}), \\
\operatorname{div} \mathbf{u}=0 .
\end{array}\right.
$$

Here $\Delta \mathbf{u}=-(\operatorname{rot})^{2} \mathbf{u}$, due to $\operatorname{div} \mathbf{u}=0$.

\footnotetext{
It should be noted that the relation (5) differs from that which was recently proposed in the work of Moffatt [9], where the similar system was analyzed by variational methods.
} 
Consider the scaling transformation (4) of (28), while $\varepsilon=R^{-1}$ :

$$
\begin{aligned}
& \varepsilon \frac{\partial}{\partial \tau}\left(\operatorname{rot}_{x y z} \mathbf{u}\right)+\varepsilon^{2} \frac{\partial}{\partial \tau}\left(\operatorname{rot}_{\xi \eta \theta} \mathbf{u}\right)+\operatorname{rot}_{x y z}\left[\mathbf{h} \times\left(\mathbf{u}-\operatorname{rot}_{x y z} \mathbf{u}\right)\right]-\varepsilon \operatorname{rot}_{x y z}\left[\mathbf{h} \times \operatorname{rot}_{\xi \eta \theta} \mathbf{u}\right] \\
&+\operatorname{rot}_{\xi \eta \theta}\left[\mathbf{h} \times\left(\mathbf{u}-\operatorname{rot}_{x y z} \mathbf{u}\right)\right]-\varepsilon^{2} \operatorname{rot}_{\xi \eta \theta}\left[\mathbf{h} \times \operatorname{rot}_{\xi \eta \theta} \mathbf{u}\right] \\
&= \varepsilon \operatorname{rot}_{x y z}\left(\Delta_{x y z} \mathbf{u}\right)+\varepsilon^{2}\left[\operatorname{rot}_{\xi \eta \theta}\left(\Delta_{x y z}\left(\Delta_{x y z} \mathbf{u}\right)\right)+2 \operatorname{rot}_{x y z}\left(\frac{\partial^{2} \mathbf{u}}{\partial x \partial \xi}+\frac{\partial^{2} \mathbf{u}}{\partial y \partial \eta}+\frac{\partial^{2} \mathbf{u}}{\partial z \partial \theta}\right)\right] \\
&+\varepsilon^{3}\left[\operatorname{rot}_{x y z}\left(\Delta_{\xi \eta \theta} \mathbf{u}\right)+2 \operatorname{rot}_{\xi \eta \theta}\left(\frac{\partial^{2} \mathbf{u}}{\partial x \partial \xi}+\frac{\partial^{2} \mathbf{u}}{\partial y \partial \eta}+\frac{\partial^{2} \mathbf{u}}{\partial z \partial \theta}\right)\right]+\varepsilon^{4}\left(\operatorname{rot}_{\xi \eta \theta}\left(\Delta_{\xi \eta \theta} \mathbf{u}\right)\right), \\
& \operatorname{div}_{x y z} \mathbf{u}=-\varepsilon \operatorname{div}_{\xi \eta \theta} \mathbf{u},
\end{aligned}
$$

where

$$
\Delta_{\xi \eta \theta} \mathbf{u}=\frac{\partial^{2} \mathbf{u}}{\partial \xi^{2}}+\frac{\partial^{2} \mathbf{u}}{\partial \eta^{2}}+\frac{\partial^{2} \mathbf{u}}{\partial \theta^{2}}
$$

We repeat the procedure of seeking the asymptotic expansion for a solution of the scaled Euler equation (5) to the scaled form of the Navier-Stokes equation (36). The integral relationship, obtained through integration of $(28)$ over $[[0,2 \pi] \times[0,2 \pi] \times$ $[0,2 \pi]]$ in $x, y$, and $z$, is

$$
\begin{aligned}
& \varepsilon \frac{\partial}{\partial \tau} \int_{0}^{2 \pi} \int_{0}^{2 \pi} \int_{0}^{2 \pi} \operatorname{rot}_{\xi \eta \theta} \mathbf{u} d x d y d z \\
& \quad+\int_{0}^{2 \pi} \int_{0}^{2 \pi} \int_{0}^{2 \pi} \operatorname{rot}_{\xi \eta \theta}\left[\mathbf{h} \times\left(\mathbf{u}-\operatorname{rot}_{x y z} \mathbf{u}\right)\right] d x d y d z \\
& \quad-\varepsilon \int_{0}^{2 \pi} \int_{0}^{2 \pi} \int_{0}^{2 \pi} \operatorname{rot}_{\xi \eta \theta}\left[\mathbf{h} \times \operatorname{rot}_{\xi \eta \theta} \mathbf{u}\right] d x d y d z \\
& =\varepsilon^{2} \int_{0}^{2 \pi} \int_{0}^{2 \pi} \int_{0}^{2 \pi} \operatorname{rot}_{\xi \eta \theta}\left(\Delta_{\xi \eta \theta} \mathbf{u}\right) d x d y d z .
\end{aligned}
$$

This is due to $2 \pi$-periodicity of $\mathbf{u}(x, y, z, \xi, \eta, \theta, \tau)$ in $x, y$, and $z$. In fact, $\varepsilon^{0}$ - and $\varepsilon^{1}$-approximations of Eq. (38) are the same as in the scaled Euler equation.

The $\varepsilon^{0}$-approximation of $(37)$ is the same as that of $(5)$ :

$$
\operatorname{rot}_{x y z}\left[\mathbf{h} \times\left(\mathbf{u}_{0}-\operatorname{rot}_{x y z} \mathbf{u}_{0}\right)\right]=0 .
$$

We seek $u_{0}$ in the form (9)

$$
\begin{aligned}
\mathbf{u}= & \alpha_{0}(\eta, \theta, \tau) \mathbf{g}_{0}+\beta(\xi, \theta, \tau) \mathbf{f}_{0}+\gamma_{0}(\xi, \eta, \tau) \mathbf{e}_{0} \\
& +\alpha_{1}(\eta, \theta, \tau) \mathbf{g}_{1}+\beta_{1}(\xi, \theta, \tau) \mathbf{f}_{1}+\gamma_{1}(\xi, \eta, \tau) \mathbf{e}_{1}+\mathbf{G}_{1},
\end{aligned}
$$

where $\mathbf{e}_{0}, \mathbf{g}_{0}, \mathbf{f}_{0}, \mathbf{e}_{1}, \mathbf{f}_{1}$ is the basis (8) of the proper subspace of the rot ryz $_{x y}$ operator, with the eigenvalue 1 . Note that all these vectors are the eigenfunctions of the Laplace operator $\Delta$ with periodic boundary conditions with the eigenvalue $(-1)$, since $\Delta=-\left(\operatorname{rot}_{x y z}\right)^{2}$.

If $A \neq 0, B \neq 0$, and $C \neq 0$

as was proved earlier.

$$
\mathbf{G}_{1} \equiv 0
$$

The $\varepsilon$-approximation of (37) yields

$$
\left.\begin{array}{l}
\frac{\partial}{\partial \tau}\left(\operatorname{rot}_{x y z} \mathbf{u}_{0}\right)-\operatorname{rot}_{x y z}\left[\mathbf{h} \times \operatorname{rot}_{\xi \eta \theta} \mathbf{u}_{0}\right]+\operatorname{rot}_{\xi \eta \theta}\left[\mathbf{h} \times\left(\mathbf{u}_{0}-\operatorname{rot}_{x y z} \mathbf{u}_{0}\right)\right] \\
-\operatorname{rot}_{x y z}\left(\Delta_{x y z} \mathbf{u}_{0}\right)=-\operatorname{rot}_{x y z}\left[\mathbf{h} \times\left(\mathbf{u}_{1}-\operatorname{rot}_{x y z} \mathbf{u}_{1}\right)\right], \\
\operatorname{div}_{x y z} \mathbf{u}_{1}=-\operatorname{div}_{\xi \eta \theta} \mathbf{u}_{0} .
\end{array}\right\}
$$


The first equation in (40) is distinguished from the first equation in (10) only by the last term on the left-hand side. Hence, in view of (17) (and instead of it), we get

$$
\begin{aligned}
& \frac{\partial \alpha_{0}}{\partial \tau}=-\alpha_{0} ; \quad \frac{\partial \beta_{0}}{\partial \tau}=-\beta_{0}, \quad \frac{\partial \gamma_{0}}{\partial \tau}=-\gamma_{0} ; \\
& \frac{\partial \alpha_{1}}{\partial \tau}=C \delta_{1}-\alpha_{1} ; \quad \frac{\partial \beta_{1}}{\partial \tau}=-B \delta_{2}-\beta_{1} ; \quad \frac{\partial \gamma_{1}}{\partial \tau}=-A \delta_{3}-\gamma_{1} .
\end{aligned}
$$

Thus, $\alpha_{0}, \beta_{0}$, and $\gamma_{0}$ are exponentially decaying in $\tau$ and so of no interest to us. As already stated, the integral relationship for $\varepsilon^{1}$-approximation remains intact when we pass from the scaled Euler equations to the scaled Navier-Stokes equation at large Reynolds numbers. Thus, Eqs. (28) are valid, since the condition $\operatorname{div}_{x y z} \mathbf{u}_{2}=$ $-\operatorname{div}_{\xi \eta \theta} \mathbf{u}_{1}$ remains intact.

Thus, the final system of equations is

$$
\begin{aligned}
& \frac{\partial \alpha_{1}}{\partial \tau}=-\alpha_{1}-C \delta_{1}, \\
& \frac{\partial \delta_{1}}{\partial \tau}=\frac{C}{2}\left(\frac{\delta^{2} \alpha_{1}}{\partial \eta^{2}}+\frac{\partial^{2} \alpha_{1}}{\partial \theta^{2}}\right), \\
& \frac{\partial \beta_{1}}{\partial \tau}=-\beta_{1}-B \delta_{2}, \\
& \frac{\partial \delta_{2}}{\partial \tau}=\frac{B}{2}\left(\frac{\delta^{2} \beta_{1}}{\partial \xi^{2}}+\frac{\partial^{2} \beta_{1}}{\partial \theta^{2}}\right), \\
& \frac{\partial \gamma_{1}}{\partial \tau}=-\gamma_{1}-A \delta_{3}, \\
& \frac{\partial \delta_{3}}{\partial \tau}=\frac{A}{2}\left(\frac{\delta^{2} \gamma_{1}}{\partial \xi^{2}}+\frac{\partial^{2} \gamma_{1}}{\partial \eta^{2}}\right) .
\end{aligned}
$$

Differentiation of the second equation in (33.1) with respect to $\tau$ and multiplication by $2 C^{-1}$ together with the application of operator $\Delta_{\xi \eta \theta}$ to the first equation and the subsequent summation of the two new equations yield

$$
\frac{2}{C} \frac{\partial^{2} \delta_{1}}{\partial \tau^{2}}=-C\left(\Delta_{\eta \theta} \delta_{1}\right)-\left(\Delta_{\eta \theta} \alpha_{1}\right)=-C\left(\Delta_{\eta \theta} \delta_{1}\right)-\frac{2}{C} \frac{\delta \partial_{1}}{\partial \tau}
$$

or

$$
\frac{\partial^{2} \delta_{1}}{\partial \tau^{2}}+\frac{\partial \delta_{1}}{\partial \tau}=-\frac{C^{2}}{2}\left(\frac{\partial^{2} \delta_{1}}{\partial \eta^{2}}+\frac{\partial^{2} \delta_{1}}{\partial \theta^{2}}\right) .
$$

Similarly

$$
\begin{aligned}
& \frac{\partial^{2} \delta_{2}}{\partial \tau^{2}}+\frac{\partial \delta_{2}}{\partial \tau}=-\frac{B^{2}}{2}\left(\frac{\partial^{2} \delta_{2}}{\partial \xi^{2}}+\frac{\partial^{2} \delta_{2}}{\partial \theta^{2}}\right) \\
& \frac{\partial^{2} \delta_{3}}{\partial \tau^{2}}+\frac{\partial \delta_{3}}{\partial \tau}=-\frac{A^{2}}{2}\left(\frac{\partial^{2} \delta_{3}}{\partial \xi^{2}}+\frac{\partial^{2} \delta_{3}}{\partial \eta^{2}}\right)
\end{aligned}
$$


Hence, the corresponding dispersion relations are

$$
\begin{aligned}
& \omega_{1}=\left(\omega_{1}+1\right)=\frac{C^{2}}{2}\left(l^{2}+m^{2}\right), \\
& \omega_{2}\left(\omega_{2}+1\right)=\frac{B^{2}}{2}\left(k^{2}+m^{2}\right), \\
& \omega_{3}\left(\omega_{3}+1\right)=\frac{A^{2}}{2}\left(k^{2}+l^{2}\right),
\end{aligned}
$$

or

$$
\begin{aligned}
& \omega_{1}=\frac{\sqrt{1+2 C^{2}\left(l^{2}+m^{2}\right)}-1}{2}>0, \\
& \omega_{2}=\frac{\sqrt{1+2 B^{2}\left(k^{2}+m^{2}\right)}-1}{2}>0, \\
& \omega_{3}=\frac{\sqrt{1+2 A^{2}\left(k^{2}+l^{2}\right)}-1}{2}>0 .
\end{aligned}
$$

Thus, if $A \neq 0, B \neq 0$, and $C \neq 0$, there are three exponentially growing solutions. As for the case of Beltrami flow, one may consider only solutions of (36) depending only on $z, \xi, \eta$, and $\tau$.

In the dimensional $\left(^{*}\right)$ form, $(45.1-45.3)$ become

$$
\begin{aligned}
& \omega_{1}^{*}=V_{0} \frac{\sqrt{1+2 R^{2} d^{2} C^{2}\left(l^{* 2}+m^{* 2}\right)}-1}{2 R d}, \\
& \omega_{2}^{*}=V_{0} \frac{\sqrt{1+2 R^{2} d^{2} B^{2}\left(k^{* 2}+m^{* 2}\right)}-1}{2 R d}-1, \\
& \omega_{3}^{*}=V_{0} \frac{\sqrt{1+2 R^{2} d^{2} A^{2}\left(l^{* 2}+m^{* 2}\right)}-1}{2 R d} .
\end{aligned}
$$

Hence

$$
\begin{aligned}
& \omega_{1}^{*} \rightarrow V_{0}|C| \sqrt{\frac{l^{* 2}+m^{* 2}}{2}}, \\
& \omega_{2}^{*} \rightarrow V_{0}|B| \sqrt{\frac{k^{* 2}+m^{* 2}}{2}}, \\
& \omega_{3}^{*} \rightarrow V_{0}|A| \sqrt{\frac{k^{* 2}+l^{* 2}}{2}},
\end{aligned}
$$

as $R \rightarrow \infty$ (cf. 31-33, and Fig. 1).

IV. Concluding remark. The undertaken study shows that at large Reynolds numbers there exists a wide class of long-wave perturbations destabilizing the basic $\mathrm{ABC}$ flows.

The proposed asymptotic analysis clearly does not apply to the perturbations, whose wave-length is comparable to period $2 \pi d$ of the basic flow. In a real situation as the wave-number $\sqrt{k^{2}+l^{2}+m^{2}}$ increases, the instability rates $\omega_{i}$ are 


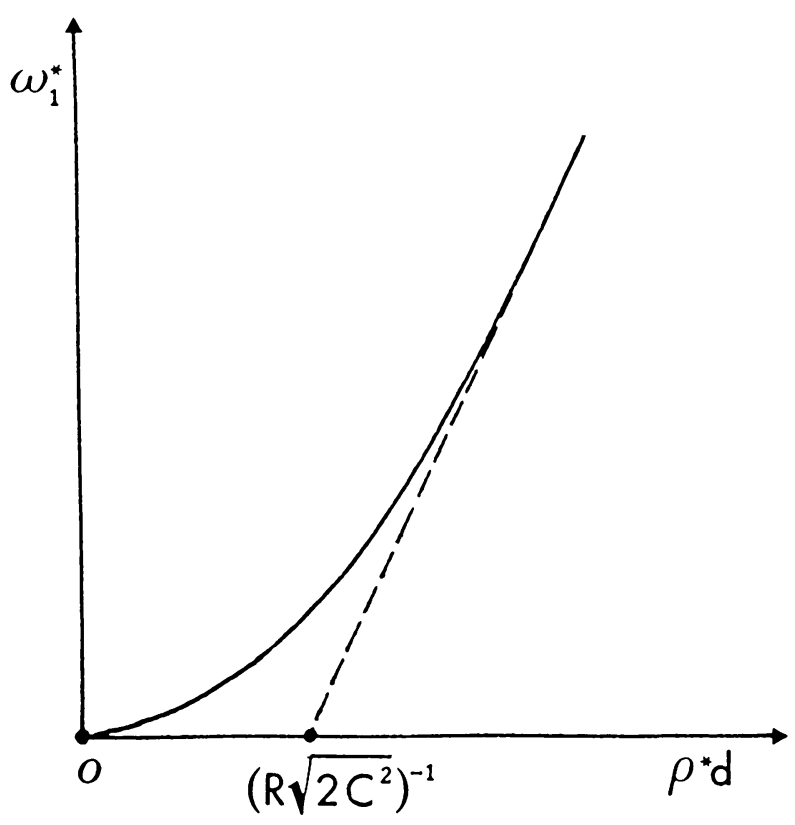

FIG. 1. Rate of instability parameter $\omega_{1}^{*}$ versus perturbation wavenumber $\rho^{*}=\sqrt{l^{* 2}+m^{* 2}}$ in region $\rho^{*} d \sim R^{-1} \ll 1$.

expected to slow down and eventually even to change sign when the perturbation wave-length becomes comparable to $2 \pi d$ (cf. Green [2]).

Finally, regarding the quasi-isotropic case $A=B=C=1$, the found transition from stability at $R \sim 1$ [12] to instability at $R \gg 1$ resembles the similar effect observed in the 2-D system of triangular eddies [18].

Acknowledgments. The authors are grateful to Professor E. Levich for helpful discussions. This research was supported in part by the U. S. Department of Energy under Grant No. DE-FG02-88ER 13822 (G.S.) and DE-FG02-88ER1387 (A.L.).

\section{REFERENCES}

[1] L. D. Meshalkin and Ya. G. Sinaì, Investigation of the stability of a stationary solution of a system of equations for the plane movement of an incompressible viscous fluid. J. Appl. Math. Mech. 25. 1700-1705 (1961)

[2] J. S. A. Green, Two-dimensional turbulence near the viscous limit. J. Fluid Mech. 62, 273-287 (1974)

[3] A. A. Nepomniashchii. On stability of secondary flows of a viscous fluid in unhounded space. $\mathbf{J}$. Appl. Math. Mech. 40, 836-841 (1976)

[4] D. N. Beaumont, The stability of spatially periodic flow's. J. Fluid Mech. 108, 461-474 (1981)

[5] K. Gotoh, M. Yamada, and J. Mizushima, The theory of stability of spatially periodic parallel flow's, J. Fluid Mech. 127, 45-58 (1983)

[6] K. Gotoh and M. Yamada. Instability of a cellular flow, J. Phys. Soc. Japan 53, 3395-3398 (1984)

[7] G. Sivashinsky and V. Yakhot. Negative viscosity phenomena in three-dimensional flows, Phys. Review A 35 (2), 810-820 (1987)

[8] G. Sivashinsky, Weak turbulence in periodic flows. Physica D 17. 243-255 (1985)

[9] H. K. Moffatt. Magnetostatic equilihria and analogous Euler flow's of arbitrary complex topology: Part 2. Stability considerations. J. Fluid Mech. 166. 359-387 (1986) 
[10] V. Yakhot and G. Sivashinsky, Negative viscosity phenomena in three-dimensional flows, Phys. Review A 35 (2), 815-820 (1987)

[11] L. Shtilman and G. Sivashinsky, Negative viscosity effect in three-dimensional flows, J. de Physique 47, 1137-1140 (1986)

[12] B. J. Bayly and V. Yakhot, Positive- and negative-effective-viscosity phenomena in isotropic and anisotropic Beltrami flows, Phys. Rev. A 34 (1), 381-391 (1986)

[13] K. Gotoh and M. Yamada, The instability of rhombic cell flow's, Fluid Dynamics Res. 1, 165-176 (1986)

[14] D. Galloway and U. Frisch, A note on the stability of a family of space-periodic Beltrami flows, J. Fluid Mech. 180, 557-564 (1987)

[15] Z. S. She, Metastability and vortex pairing in the Kolmogorov flow, Phys. Lett. A 124, 161-164 (1987)

[16] U. Frisch, Z. S. She, and P.-L. Sulem, Large-scale flow driven by the anisotropic kinetic alpha effect, Physica D 28, 382-392 (1987)

[17] A. Libin, G. I. Sivashinsky, and E. Levich, Long-wave instability of periodic flows at large Reynolds numbers, Phys. Fluids 30, 2984-2986 (1987)

[18] A. Libin and G. I. Sivashinsky, Long wavelength instability of triangular eddies at large Reynolds numbers, Phys. Lett. A 144, 172-178 (1990) 